\title{
Enrichment of Poultry Diets with Polyunsaturated Fatty Acids (PUFA) for Human Consumption
}

\author{
Hanan Al-Khalifa* \\ Kuwait Institute for Scientific Research, Kuwait \\ *Corresponding author: Hanan Al-Khalifa, PO Box 24885, 13109 Safat, Kuwait
}

Submission: 眥 August 30, 2017; Published: 眥 November 14, 2017

Abbreviations: PUFA: Polyunsaturated Fatty Acids; EPA: Eicosapentaenoic Acid; DHA: Docosahexaenoic Acid; AA: Arachidonic Acid; $\alpha$-LNA: $\alpha$-Linolenic Acid

\section{Introduction}

In recent years, polyunsaturated fatty acids (PUFA) have received considerable attention in both human and animal nutrition, particularly those of the $n-3$ family. These are PUFA in which the first double bond is situated on the third carbon atom from the methyl end of the fatty acid molecule. Consumption of n-3 PUFA are low, particularly the long chain ( $>18$ carbon atoms) ones that are most commonly found in fish oils. As a mean of increasing the low consumption of the long chain n-3 PUFA by humans consuming western diets, there has been some interest in the enrichment of poultry meat with these fatty acids for people seeking healthy lifestyles.

\section{Involvement in Health and Clinical Problems}

Vitality of living cells depends profoundly on dietary lipids that are incorporated into phospholipid layers of cellular membranes. n-3 PUFA, eicosapentaenoic acid (EPA) and docosahexaenoic acid (DHA), are reported to compete with arachidonic acid (AA) for this incorporation. Since AA is responsible for up-regulation of eicosanoids such as leukotrienes, this competitive inhibition downregulates inflammation responses related to many diseases and disorders such as cardiovascular disease, increased triglycerides, blood pressure, thrombosis, atherosclerosis, stress, mental problems, asthma and rheumatoid arthritis. These benefits of an optimal ratio of n-3/n-6 PUFAs on health are just a few examples of a wide range of clinical problems that are improved by consumption of the very long chain n-3 fatty acids [1-4].

\section{n-3PUFA enrichment of poultry diet}

Traditionally, fish and fish oil are the main sources of essential, long chain n-3 PUFA that induce modifications in the lipid composition of poultry products because marine sources in general contain high levels of EPA and DHA PUFA. Of less nutritional importance are plant sources such as linseed that is rich in $\alpha$-linolenic acid ( $\alpha$-LNA). $\alpha$-LNA is an 18 carbon n-3 fatty acid that is the precursor to the long chain n-3 PUFA, but because the efficiency of conversion is so low in humans, the accumulation of $\alpha$-LNA is of little real nutritional benefit. Chickens fed linseed oil deposit significant amounts of $\alpha$-LNA in the egg yolk (in laying hens) and meat (in broilers) [5-9]. However, to achieve enrichment of poultry products with long chain n-3 PUFA, marine oils need to be fed. Bou et al. [10] reported that supplementing broiler diets with $25 \mathrm{~g} / \mathrm{kg}$ fish oil produced double the amount of beneficial EPA and DHA than diets supplied with $12.5 \mathrm{~g} / \mathrm{kg}$ fish oil. High concentrations of fish oil supplementation decrease the saturated and mono saturated FA and increase the very long chain PUFA in poultry meat [11]. Lopez-Ferrer et al. [12] substituted $82 \mathrm{~g} / \mathrm{kg}$ fish oil in supplemented broilers diet with the same amount of linseed and rapeseed. They concluded that the total amount of the very long chain PUFA in the chicken meat decreased when fish oil was removed from the diet. On the contrary, the n-6 PUFA and mono saturated FA in form of oleic acid increased. These results support the fact that fish oil is more efficient in elevating levels of long chain n-3 PUFA.

\section{Involvement in Avian Immune Function}

The immunomodulatory effect of PUFA in broiler chickens occurs by affecting intercellular communications and signals that change the reactivity of leukocytes upon antigenic stimulation. This effect is highly associated with down-regulation or up-regulation of different cytokines that are believed to affect the avian immune function such as IL-1 $\beta$, IFN- $\gamma$, MGF, IL-1, IL-4, IL-2 [13,14].

There is some concern that diets enriched with n-3 PUFA have detrimental effects on chicken immunity and impair resistance to infection. However, it is not clear whether this concern is justified, since some studies show no effect [15], some show a detrimental effect [16] while some show an improvement $[16,17]$ in chicken immune response following feeding of n-3 PUFA. 
All in all, n-3 PUFA are essential for human health and enrichment of these fatty acids is successful. However, care should be taken because these fatty acids may modulate the immune system.

\section{References}

1. Doughman S, Ryan A, Krupanidhi S, Sanjeevi C, Mohan V (2013) High DHA dosage from oil improves postprandial hypertriglyceridemia and is safe for type-2 diabetics. Int J Diabetes Dev Ctries 33: 75-82.

2. Simopoulos AP (2016) An increase in the $\omega-6 / \omega-3$ fatty acid ratio increases the risk for obesity. Nutrients 8(3): 128-138.

3. Karakas S, Perroud B, Kind T, Palazoglu M, Fiehn O (2016) Changes in plasma metabolites and glucose homeostasis during omega-3 polyunsaturated fatty acid supplementation in women with polycystic ovary syndrome. BBA Clin 5: 179-185.

4. Derosa G, Cicero AF, D Angelo A, Borghi C, Maffioli P (2016) Effects of $\mathrm{n}-3$ pufas on fasting plasma glucose and insulin resistance in patients with impaired fasting glucose or impaired glucose tolerance. Biofactors 42(3): 316-322.

5. Al-Khalifa H (2015) Production of added-value poultry meat: Enrichment with n-3 polyunsaturated fatty acids. World's Poultry Science Journal 71(2): 319-326.

6. Al-Khalifa HA, Al-Nasser M, Al-Bahouh G, Ragheb S, Al-Qalaf N, et al. (2016) The effect of polyunsaturated fatty acids on avian immune cell subpopulations in peripheral blood, spleen, and thymus. World's Poultry Science Journal 72: 531-534

7. Ahmad S, Kamran Z, KoutoulisK C (2016) Supplemental linseed on egg production. In: Hester PY (Eds.), Egg Innovation and Strategies for Improvement, Academic Press, San Diego, California, United States, pp. 349-364.

8. Apperson KD, Cherian G (2017) Effect of whole flax seed and carbohydrase enzymes on gastrointestinal morphology, muscle fatty acids, and production performance in broiler chickens. Poultry Science 96(5): 1228-1234.

9. Beheshti MH, Shehab A, Cherian G (2017) Methionine supplementation augments tissue n-3 fatty acid and tocopherol content in broiler birds fed flaxseed. Animal Feed Science and Technology 228: 149-158.

10. Bou R, Guardiola F, Tres A, Barroeta AC, Codony R (2004) Effect of dietary fish oil, alpha-tocopherl acetate, and zinc supplementation on the composition and consumer acceptability of chicken meat. Poult Sci 83(2): 282-292.

11. Lopez-FS, Baucells MD, Barroeta AC, Grashorn MA (2001b) n-3 Enrichment of chicken meat. 1. Use of very long-chain fatty acids in chicken diets and their influence on meat quality: fish oil. Poultry Science 80(6): 741-752.

12. Lopez-FS, Baucells MD, Barroeta AC, Grashorn MA (1999b) n-3 enrichment of chicken meat using fish oil: alternative substituation with rapeseed and linseed oils. Poult Sci 78(3): 356-365.

13. Al-Khalifa H (2012) Algal Biomass Enriches Poultry Meat with n-3 PUFA and Enhances Immune Response. Journal of Petroleum and Environmental Biotechnology 3(7): 28. Proceedings of the OMICS Group Conference, Global \& Bio-products Summit, San Antonio, USA.

14. Jalali S, Rabiei R, Kheiri F (2015) Effects of dietary soybean and sunflower oils with and without L-carnitine supplementation on growth performance and blood biochemical parameters of broiler chicks. Archiv fuer Tierzucht 58: 387-394.

15. Puthpongsiriporn U, Scheideler SE (2005) Effects of dietary ratio of linoleic to linolenic acid on performance, antibody production, and in vitro lymphocyte proliferation in two strains of Leghorn pullet chicks. Poultry Science 84(6): 846-857.

16. Al-Khalifa H, Givens DI, Rymer C, Yaqoob P (2012) Effect of n-3 fatty acids on immune function in broiler chickens. Poultry Science 91(1): 74-88.

17. Yang X, Yuming G (2006) Modulation of intestinal mucosal immunity by dietary polyunsaturated fatty acids in chickens. Food and Agricultural Immunology 17(2): 129-137. 